\title{
Schmidt Decomposition and Coherence of Interfering Alternatives
}

\author{
D. V. Fastovets ${ }^{a, *}$, Yu. I. Bogdanov ${ }^{a, * *}$, N. A. Bogdanova ${ }^{a}$, and V. F. Lukichev ${ }^{a}$ \\ ${ }^{a}$ Valiev Institute of Physics and Technology, Russian Academy of Sciences, Moscow, 117218 Russia. \\ *e-mail:fast93@mail.ru \\ **e-mail: bogdanov_yurii@inbox.ru
}

Received February 3, 2021; revised February 27, 2021; accepted March 1, 2021

\begin{abstract}
The Schmidt decomposition and the correlational analysis based on it make it possible to identify statistical dependences between various subsystems of a single physical system. The systems under consideration can be both quantum states and classical probability distributions. In this study, two different physical systems are considered: quantum Schrödinger cat states and double-slit interference of microparticles. It is shown that the considered systems have a single internal structure and can be described in general terms of interfering alternatives. An effective approach is developed that allows us to calculate optical characteristics of interference such as visibility and coherence. It is shown that the scalar product of the states of the environment of interfering alternatives acts as a natural generalization of the classical complex parameter of the coherence of light oscillations, which determines the visibility of the interference pattern. A simple quantitative relationship is obtained between the visibility of the interference pattern and the Schmidt number, which determines the level of connection between a quantum system and its environment. The developed approaches are generalized to the case of multidimensional Schrödinger cat states.
\end{abstract}

DOI: $10.1134 / \mathrm{S} 1063739721040065$

\section{INTRODUCTION}

The interference of quantum states is one of the cornerstones of the concept of quantum computing [1,2]. The use of this effect in combination with entanglement and quantum parallelism makes it possible to effectively solve various problems that are inaccessible to a classical computer. Interference effects are manifested in a variety of systems, such as a diffraction grating, biphoton fields, and a two-beam electron interferometer [3]. It is also important to note the interference effects that manifest themselves in quantum Schrödiger cat states, which are a superposition of coherent states [4] and are actively used in quantum optics [57]. Schrödinger's cat states are of great interest in quantum communications and quantum optics and are used in various fields, such as quantum computing in continuous variables [8-10], quantum error correction codes [11, 12], and precision measurements [13, 14]. These practical applications explain the rapid development of the theory of quantum correlations in bipartite quantum states. The generalization of twomode superpositions to coherent states with more than two degrees of freedom is quite simple, but it contains a number of hidden properties [15, 16], including those related to multipartite entanglement, which is the most valuable resource for solving communication and computational problems. Multimode entangled coherent states can serve as a resource for the implementation of GHZ and W states [17], as well as cluster states [18]. A method for calculating the coherence of interfering quantum systems has been developed. The proposed approach is based on the formalism of Schmidt correlations [19] and allows us to assess the degree of interconnection between two different subsystems. The relationship between the quantum Schrödiger cat states and quantum interference in a double-slit experiment is investigated.

A method has been developed that makes it possible to efficiently calculate the optical characteristics of interference such as visibility and coherence. The proposed method is generalized to the case of multidimensional Schrödiger cat states. In this generalization, analytical formulas are obtained that make it possible to model and calculate the arising interference patterns and characteristics. The results obtained can be used in the development of high-dimensional quantum information processing systems.

\section{SCHMIDT DECOMPOSITION}

The mathematical apparatus related to the Schmidt decomposition provides a convenient tool that allows us to analyze the entanglement of quantum states and correlations in classical distributions [20]. Assume $|\psi\rangle$ is the quantum state of a bipartite system consisting of subsystems $A$ and $B$. Then, the Schmidt decomposition is given in the form [2]: 


$$
|\psi\rangle=\sum_{k=1}^{s} \sqrt{\lambda_{k}}\left|\psi_{k}^{A}\right\rangle\left|\psi_{k}^{B}\right\rangle
$$

where $\lambda_{k}$ are the Schmidt weight coefficients, sorted in descending (nonincreasing) order, $\left|\psi_{k}^{A}\right\rangle$ and $\left|\psi_{k}^{B}\right\rangle$ are the corresponding Schmidt modes of subsystems $A$ and $B$, and $s$ is the dimension of the smallest of the subsystems, $s=\min (\operatorname{dim} A, \operatorname{dim} B)$. Based on the set of Schmidt coefficients, a characteristic is introduced that describes the effective number of modes: the Schmidt number:

$$
K=\frac{1}{\sum_{k} \lambda_{k}^{2}} .
$$

The Schmidt number lies on the segment $[1, s]$ and allows us to assess the degree of interconnection between two subsystems: $K=1$ corresponds to only one nonzero term in the decomposition (1) and hence the absence of correlation and quantum entanglement; and $K=s$ corresponds to the maximal correlation and entanglement between subsystems.

The considered mathematical toolkit, in combination with the root approach, allows us to analyze probability distributions and perform statistical data analysis $[19,21]$.

\section{COHERENCE OF INTERFERING ALTERNATIVES}

An important case of using the Schmidt decomposition (1) corresponds to the situation when the environment of the system under consideration $A$ acts as subsystem $B$. Considering the entanglement between the quantum system and its environment sheds light on the nature of the coherence of interfering quantum states.

Let us consider this case in more detail. Let there be two interfering alternatives $\left|\varphi_{1}\right\rangle$ and $\left|\varphi_{2}\right\rangle$, entangled with the environment's states $\left|e_{1}\right\rangle$ and $\left|e_{2}\right\rangle$, respectively (we assume that all indicated states are unity normalized):

$$
|\psi\rangle=\frac{1}{\sqrt{2+q_{1} q_{2}+q_{1}^{*} q_{2}^{*}}}\left(\left|\varphi_{1}, e_{1}\right\rangle+\left|\varphi_{2}, e_{2}\right\rangle\right),
$$

where $q_{1}=\left\langle\varphi_{1} \mid \varphi_{2}\right\rangle$ is the amplitude of the probability of observing an alternative $\left|\varphi_{1}\right\rangle$ provided that an alternative $\left|\varphi_{2}\right\rangle$ has been prepared. Likewise $q_{2}=\left\langle e_{1} \mid e_{2}\right\rangle$ is the amplitude of the probability of coincidence of the states of the environment $\left|e_{1}\right\rangle$ and $\left|e_{2}\right\rangle$. The special case when the alternatives are perfectly distinguishable $\left(q_{1}=0\right)$ corresponds to the results presented in [22, 23].

The problem under consideration is reduced to the study of a two-qubit system, regardless of the complexity of the interfering states and the states of the environment themselves. In this case, the first qubit is set by the interfering alternatives; and the second, by the corresponding states of the environment. By orthogonalization, it is easy to obtain the basis states of the qubits under consideration.

For a qubit related to interfering alternatives, we obtain the following states of logical zero and logical one:

$$
|0\rangle_{1}=\left|\varphi_{1}\right\rangle, \quad|1\rangle_{1}=\frac{1}{\sqrt{1-\left|q_{1}\right|^{2}}}\left(\left|\varphi_{2}\right\rangle-q_{1}\left|\varphi_{1}\right\rangle\right) .
$$

Similarly, for the environment's qubit, we have

$$
|0\rangle_{2}=\left|e_{1}\right\rangle, \quad|1\rangle_{2}=\frac{1}{\sqrt{1-\left|q_{2}\right|^{2}}}\left(\left|e_{2}\right\rangle-q_{2}\left|e_{1}\right\rangle\right) \text {. }
$$

As a result, the two-qubit state (3) can be represented as

$$
|\psi\rangle=c_{00}|00\rangle+c_{01}|01\rangle+c_{10}|10\rangle+c_{11}|11\rangle,
$$

where

$$
\begin{gathered}
c_{00}=\frac{1+q_{1} q_{2}}{\sqrt{2+q_{1} q_{2}+q_{1}^{*} q_{2}^{*}}}, \quad c_{01}=\frac{q_{1} \sqrt{1-\left|q_{2}\right|^{2}}}{\sqrt{2+q_{1} q_{2}+q_{1}^{*} q_{2}^{*}}}, \\
c_{10}=\frac{q_{2} \sqrt{1-\left|q_{1}\right|^{2}}}{\sqrt{2+q_{1} q_{2}+q_{1}^{*} q_{2}^{*}}}, \quad c_{11}=\frac{\sqrt{\left(1-\left|q_{1}\right|^{2}\right)\left(1-\left|q_{2}\right|^{2}\right)}}{\sqrt{2+q_{1} q_{2}+q_{1}^{*} q_{2}^{*}}} .
\end{gathered}
$$

It is easy to show [23] that the following formula is valid for the Schmidt number:

$$
K=\frac{1}{1-2 \Delta},
$$

where $\Delta=\left|c_{00} c_{11}-c_{01} c_{10}\right|^{2}$.

From the presented expressions it follows that the square of the modulus of the determinant is

$$
\Delta=\frac{\left(1-\left|q_{1}\right|^{2}\right)\left(1-\left|q_{2}\right|^{2}\right)}{\left(2+q_{1} q_{2}+q_{1}^{*} q_{2}^{*}\right)^{2}}
$$

Note that in the case of clearly distinguishable alternatives, for example, for narrow slits in Young's experiment $\left(q_{1}=0\right)$, we get $\Delta=\left(1-\left|q_{2}\right|^{2}\right) / 4$. All the other basic quantities can be expressed through values $\Delta$ and $K$. For example, the Schmidt weights will be

$$
\lambda_{0}=\frac{1}{2}\left(1+\sqrt{\frac{2-K}{K}}\right)=\frac{1}{2}(1+\sqrt{1-4 \Delta}), \quad \lambda_{1}=\frac{1}{2}\left(1-\sqrt{\frac{2-K}{K}}\right)=\frac{1}{2}(1-\sqrt{1-4 \Delta})
$$


In the theory of optical phenomena, the concept of visibility of an interference pattern $V$ is inextricably linked with the phenomenon of interference [24]. This parameter characterizes the intensity modulation by interference fringes. Visibility ranges from 0 to 1 . A visibility value of zero corresponds to a uniformly illuminated screen and therefore no interference pattern. A visibility value of one indicates a contrast and clarity of the interference pattern on the screen. The visibility of the interference pattern (for narrow slits) is determined in classical optics by the formula [24]

$$
V=\frac{I_{\max }-I_{\min }}{I_{\max }+I_{\min }} .
$$

Here $I_{\max }$ and $I_{\min }$ are the maximal and minimal intensity of the recorded optical signal. In the language of the Schmidt decomposition, the entire $\lambda_{0}$ of the main (zero) mode acts as a useful signal $I_{\max }$; and the entire $\lambda_{1}$ of the first mode, as noise $I_{\min }$; therefore, we obtain the following relationship between visibility and the Schmidt number [22]:

$$
V=\lambda_{0}-\lambda_{1}=\sqrt{\frac{2-K}{K}}=\sqrt{1-4 \Delta} .
$$

Note that a direct examination of the interference pattern from two narrow slits [22] shows that our defi- nition of visibility (9) fully agrees with the classical definition. For clearly distinguishable alternatives (for narrow slits in Young's experiment), when $q_{1}=0$, we have the following simple relationship between the visibility and coherence of the environment's states:

$$
V=\left|q_{2}\right|=\left|\left\langle e_{1} \mid e_{2}\right\rangle\right| .
$$

Comparing the presented description of interfering quantum alternatives with the classical description of the phenomenon of coherence, we see that the scalar product of the states of the environment $q_{2}=\left\langle e_{1} \mid e_{2}\right\rangle$ acts as a natural generalization of the classical complex parameter $\gamma$, called the degree of coherence of light oscillations [25].

The developed mathematical apparatus can be applied to any system in which there is an interference of two different alternatives. In this study, we consider the multimode Schrödiger cat states and their relationship with the double-slit experiment (Sections 4 and 5).

For the sake of completeness, let us also present a generalization of the obtained formulas to the case when the amplitudes of alternatives $f_{1}$ and $f_{2}$ differ. Instead of formula (3), we get

$$
|\psi\rangle=\frac{1}{\sqrt{\left|f_{1}\right|^{2}+\left|f_{2}\right|^{2}+f_{1}^{*} f_{2} q_{1} q_{2}+f_{1} f_{2}^{*} q_{1}^{*} q_{2}^{*}}}\left(f_{1}\left|\varphi_{1}, e_{1}\right\rangle+f_{2}\left|\varphi_{2}, e_{2}\right\rangle\right) .
$$

In this case, the amplitudes of the probabilities of the basis states will be given by the following formulas:

$$
\begin{gathered}
c_{00}=\frac{f_{1}+f_{2} q_{1} q_{2}}{\sqrt{\left|f_{1}\right|^{2}+\left|f_{2}\right|^{2}+f_{1}^{*} f_{2} q_{1} q_{2}+f_{1} f_{2}^{*} q_{1}^{*} q_{2}^{*}}}, \quad c_{01}=\frac{f_{2} q_{1} \sqrt{1-\left|q_{2}\right|^{2}}}{\sqrt{\left|f_{1}\right|^{2}+\left|f_{2}\right|^{2}+f_{1}^{*} f_{2} q_{1} q_{2}+f_{1} f_{2}^{*} q_{1}^{*} q_{2}^{*}}}, \\
c_{10}=\frac{f_{2} q_{2} \sqrt{1-\left|q_{1}\right|^{2}}}{\sqrt{\left|f_{1}\right|^{2}+\left|f_{2}\right|^{2}+f_{1}^{*} f_{2} q_{1} q_{2}+f_{1} f_{2}^{*} q_{1}^{*} q_{2}^{*}}}, \quad c_{11}=\frac{f_{2} \sqrt{\left(1-\left|q_{1}\right|^{2}\right)\left(1-\left|q_{2}\right|^{2}\right)}}{\sqrt{\left|f_{1}\right|^{2}+\left|f_{2}\right|^{2}+f_{1}^{*} f_{2} q_{1} q_{2}+f_{1} f_{2}^{*} q_{1}^{*} q_{2}^{*}}} .
\end{gathered}
$$

The square of the modulus of the determinant is now

$$
\Delta=\frac{\left|f_{1}\right|^{2}\left|f_{2}\right|^{2}\left(1-\left|q_{1}\right|^{2}\right)\left(1-\left|q_{2}\right|^{2}\right)}{\left(\left|f_{1}\right|^{2}+\left|f_{2}\right|^{2}+f_{1}^{*} f_{2} q_{1} q_{2}+f_{1} f_{2}^{*} q_{1}^{*} q_{2}^{*}\right)^{2}} .
$$

Note that in the case when $f_{1}=f_{2}=1$, we get the theory outlined above. Note also that $f_{1}$ and $f_{2}$ are relative amplitudes. All the presented formulas remain invariant when the amplitudes are multiplied by an arbitrary complex constant $C: f_{1} \rightarrow C f_{1}, f_{2} \rightarrow C f_{2}$ (in this case, the phase of number $C$ will lead to the multiplication of both amplitudes of the state by an insignificant identical phase factor).

\section{THE TWO-MODE QUANTUM SCHRÖDINGER CAT STATE}

The Schrödiger cat state is a superposition of coherent states differing in phase by $\pi$ (see, for example, $[26,27])$ :

$$
\left|c a t_{\alpha}\right\rangle=\frac{1}{\sqrt{2+2 q_{\alpha}}}(|\alpha\rangle+|-\alpha\rangle)
$$



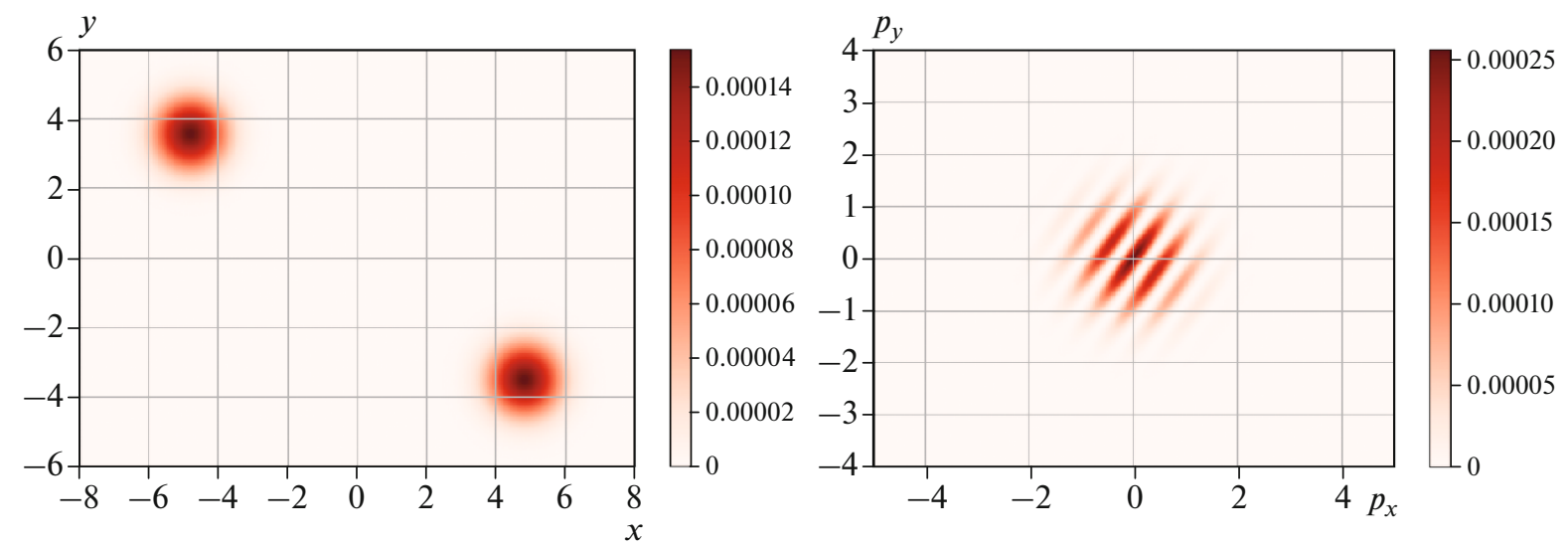

Fig. 1. Probability distribution at $\alpha=3.4, \beta=-2.5$. The coordinate distribution is on the left side. The momentum distribution is on the right side.

where $q_{\alpha}=\langle\alpha \mid-\alpha\rangle=\exp \left(-2|\alpha|^{2}\right)$.

The multimode states formed by several subsystems (modes) are of significant practical interest. The presence of entanglement in such states makes them a universal tool for use in various quantum algorithms [28]. The simplest state of this kind, representing a system of two entangled coherent states, is the twomode Schrödiger cat state [29]:

$$
\left|c a t_{\alpha \beta}\right\rangle=\frac{1}{\sqrt{2+2 q_{\alpha} q_{\beta}}}(|\alpha, \beta\rangle+|-\alpha,-\beta\rangle)
$$

It is convenient to visualize the two-mode Schrödiger cat state when using the coordinate and momentum representations of the wave function (see Fig. 1). The wave function in the coordinate representation has the form (the insignificant phase factor is omitted):

$$
\psi_{\alpha \beta}(x, y)=\frac{\sqrt{2} C_{\alpha \beta}}{\sqrt{\pi}} \exp \left(-\frac{x^{2}+y^{2}}{2}\right) \cosh ((\alpha x+\beta y) \sqrt{2}),
$$

where $C_{\alpha \beta}=\left(\exp \left(2 \bar{\alpha}^{2}+2 \bar{\beta}^{2}\right)+\exp \left(-2 \overline{\bar{\alpha}}^{2}-2 \overline{\bar{\beta}}^{2}\right)\right)^{-1 / 2}$ is the normalization factor, in which the following designations are introduced for the real and imaginary parts of the amplitudes of the coherent states: $\bar{\alpha}=\operatorname{Re}(\alpha)$, $\overline{\bar{\alpha}}=\operatorname{Im}(\alpha), \bar{\beta}=\operatorname{Re}(\beta)$, and $\overline{\bar{\beta}}=\operatorname{Im}(\beta)$.

The presented formula is a direct consequence of formula (12) without taking into account the insignificant phase factor. The Fourier transform of this function corresponds to the state wave function (12) in the momentum representation:

$$
\tilde{\psi}_{\alpha \beta}\left(p_{x}, p_{y}\right)=\frac{\sqrt{2} \tilde{C}_{\alpha \beta}}{\sqrt{\pi}} \exp \left(-\frac{p_{x}^{2}+p_{y}^{2}}{2}\right) \cos \left(\left(\alpha p_{x}+\beta p_{y}\right) \sqrt{2}\right),
$$

where $\tilde{C}_{\alpha \beta}=\left(\exp \left(2 \overline{\bar{\alpha}}^{2}+2 \overline{\bar{\beta}}^{2}\right)+\exp \left(-2 \bar{\alpha}^{2}-2 \bar{\beta}^{2}\right)\right)^{-1 / 2}$ is the normalization factor. We can see that the constants $C_{\alpha \beta}$ and $\tilde{C}_{\alpha \beta}$ are transformed into each other if $\bar{\alpha}^{2}$ and $\overline{\bar{\alpha}}^{2}$ (and $\bar{\beta}^{2}$ and $\overline{\bar{\beta}}^{2}$ ) are swapped. In more detail, when the coherent state is rotated through angle $\pi / 2$, the coordinate transforms into momentum $x \rightarrow p$, and amplitude $\alpha_{j}$ transforms into a new amplitude $\alpha_{j}^{\prime}: \alpha_{j} \rightarrow \alpha_{j}^{\prime}=\exp (i \pi / 2) \alpha_{j}=i \alpha_{j}$; therefore, $\bar{\alpha}_{j}^{\prime}=-\overline{\bar{\alpha}}_{j}$ and $\overline{\bar{\alpha}}_{j}^{\prime}=\bar{\alpha}_{j}$. With this transformation, the coordinate wave function (13) is transformed into a momentum wave function (14).

In Fig. 1 the interference fringes are clearly visible in the momentum representation.

Since this system contains interfering alternatives, the general approaches presented in Sections 2 and 3 are applicable to study the quantum correlation (entanglement) between modes, as well as the phenomena of coherence and interference. Now it should be assumed that $q_{1}=q_{\alpha}=\langle\alpha \mid-\alpha\rangle=\exp \left(-2|\alpha|^{2}\right)$, and $q_{2}=q_{\beta}=\langle\beta \mid-\beta\rangle=\exp \left(-2|\beta|^{2}\right)$. 

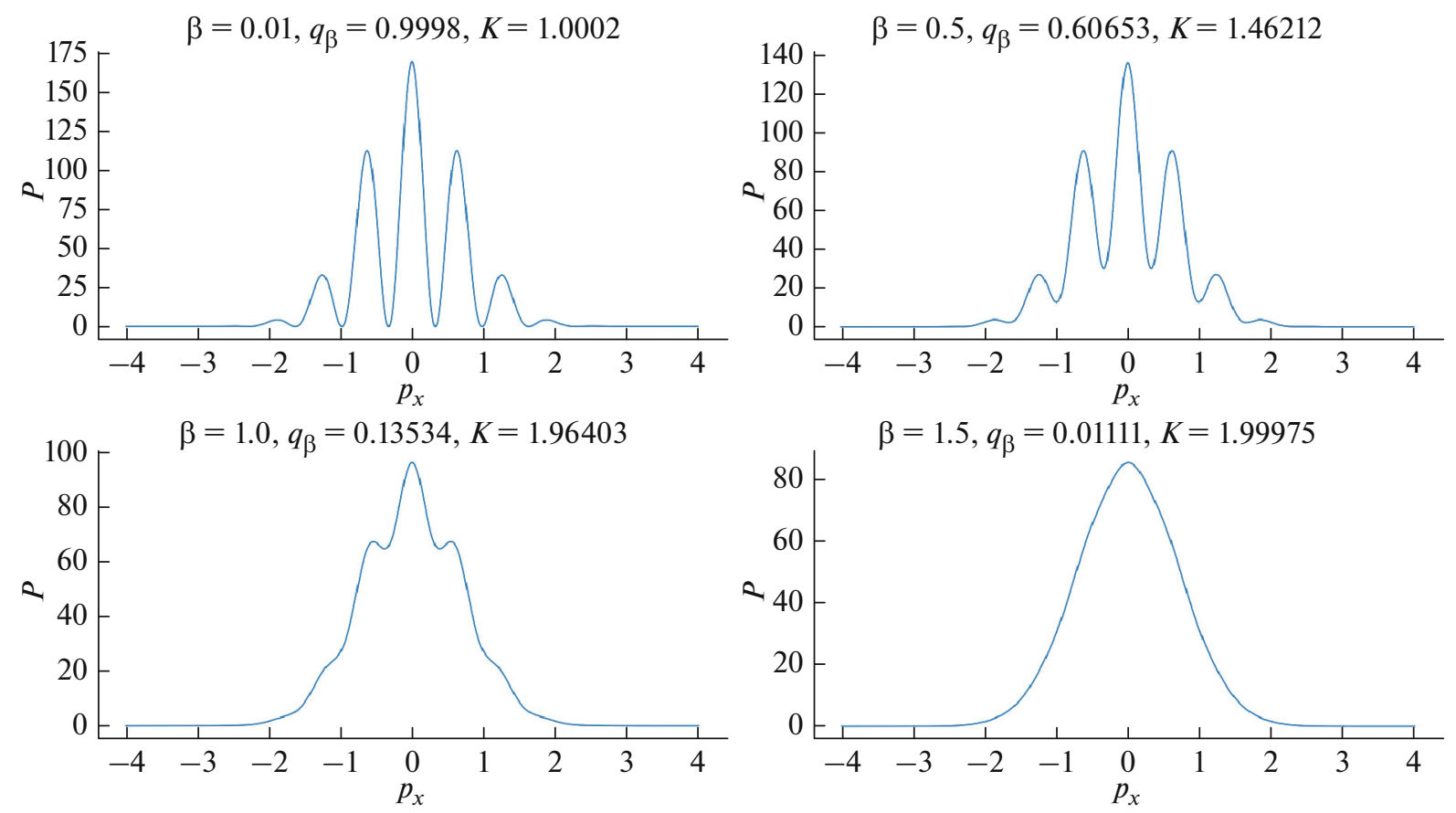

Fig. 2. Illustration of the disappearance of the interference pattern with increasing parameter $\beta(\alpha=3.4)$.

To demonstrate how the gradual disappearance of the interference pattern (and the decrease in coherence) occurs, we fix the value of parameter $\alpha$ and we will vary the value of parameter $\beta$. Figure 2 shows the corresponding interference patterns formed by the marginal momentum distribution $P\left(p_{x}\right)=\int\left|\tilde{\Psi}_{\alpha \beta}\left(p_{x}, p_{y}\right)\right|^{2} d p_{y}$ momentum of the first mode.

When calculating these dependences, the second subsystem (represented by parameter $\beta$ ) plays the role of the environment of the main system represented by parameter $\alpha$. Thus, the introduced parameter $q_{\beta}=\langle\beta \mid-\beta\rangle$ has the meaning of coherence (or visibility, if we take into account that $q_{\beta}$ is a real, positive number, and $q_{\alpha}=\exp \left(-2|\alpha|^{2}\right)$ is small). Note that as parameter $\beta$ grows the visibility of the interference pat- tern decreases (which is also confirmed by the shape of the presented plots) and the Schmidt number increases, which indicates an increase in the correlation between the system and its environment.

It is also worth noting the possibility of transition from Schrödiger cat states to the double-slit experiment and back. This is possible due to the two-level nature of these systems, which is similar from a physical point of view.

The double-slit experiment is one of the most important examples of interference in which the fundamental principles of quantum mechanics are manifested [30].

As the simplest model of a double-slit experiment, we consider a model defined by the superposition of two two-dimensional Gaussian probability amplitudes:

$$
\psi(x, y)=\frac{\tilde{C}_{a b}}{\sqrt{2}} \frac{1}{\sqrt{2 \pi \sigma_{x} \sigma_{y}}}\left[\exp \left(-\frac{(x-a)^{2}}{4 \sigma_{x}^{2}}-\frac{(y-b)^{2}}{4 \sigma_{y}^{2}}\right)+\exp \left(-\frac{(x+a)^{2}}{4 \sigma_{x}^{2}}-\frac{(y+b)^{2}}{4 \sigma_{y}^{2}}\right)\right],
$$

where

$$
\tilde{C}_{a b}=\left(\exp \left(\frac{\bar{a}^{2}}{2 \sigma_{x}^{2}}+\frac{\overline{\bar{b}}^{2}}{2 \sigma_{y}^{2}}\right)+\exp \left(-\frac{\bar{a}^{2}}{2 \sigma_{x}^{2}}-\frac{\bar{b}^{2}}{2 \sigma_{y}^{2}}\right)\right)^{-1 / 2}
$$

is the normalization constant, $\bar{a}=\operatorname{Re}(a)$, $\overline{\bar{a}}=\operatorname{Im}(a), \bar{b}=\operatorname{Re}(b)$, and $\overline{\bar{b}}=\operatorname{Im}(b)$.
In the presented formula, variable $x$ corresponds to the coordinate of the interfering microparticle, and variable $y$ corresponds to the environment (the read- 
ings of the detector or the coordinate of the detecting particle). In the case of real parameters $a$ and $b$, we can give the following visual interpretation of formula (15). The interfering particle is described by two Gaussian distributions with mean $\pm a$ and variance $\sigma_{x}^{2}$. Similarly, detection is described by two Gaussian distributions with mean $\pm b$ and variance $\sigma_{y}^{2}$. When a particle passes through a slit centered at the point $x=+a$, the detector fixes parameter $y$ (the coordinate of the detecting particle) near $y=+b$. Similarly, when a particle passes through a slit centered at the point $x=-a$, the detector fixes parameter $y$ near $y=-b$. Thus, the readings of the detector are correlated with the position of the particle. From the quantum mechanical point of view, the state under consideration is entangled: coordinates $x$ and $y$ are correlated (entangled) with each other. It is obvious that the correlation between $x$ and $y$ is high when $b \gg \sigma_{y}$. In this case, however, the interference pattern disappears.

Based on the presented expression (15) for the coordinate wave function, by the simple calculations performed in [22], it is possible to obtain a twodimensional wave function in the momentum representation by the Fourier transform

$$
\tilde{\psi}\left(p_{x}, p_{y}\right)=\frac{\sqrt{2} \tilde{C}_{a b}}{\sqrt{\pi}} \sqrt{\sigma_{x} \sigma_{y}} \exp \left(-\sigma_{x}^{2} p_{x}^{2}-\sigma_{y}^{2} p_{y}^{2}\right) \cos \left(p_{x} a+p_{y} b\right) .
$$

Comparing the structure of this formula with formula (14), we can see clear analogies. The presence of interference behavior in both formulas leads to the conclusion that a transition from one representation (two-mode Schrödiger cat state) to another (doubleslit interference of the wave function) is possible. Full compliance of these formulas is observed at $a=\alpha \sqrt{2}$, $b=\beta \sqrt{2}, \sigma_{x}=\sigma_{y}=\frac{1}{\sqrt{2}}$, and $\tilde{C}_{a b}=\tilde{C}_{\alpha \beta}$.

The found close connection between quantum interference and the mathematical apparatus of quan- tum two-dimensional Schrödiger cat states makes it possible to effectively estimate the optical parameters of the interference systems.

\section{GENERALIZATION TO THE MULTIMODE CASE}

By analogy with the two-mode Schrödiger cat state, we can introduce a state consisting of an arbitrary number of modes. The Schrödiger cat state of dimension $n$ is defined by the formula

$$
\left|c a t_{\alpha_{1} \alpha_{2} \ldots \alpha_{n}}\right\rangle=\frac{1}{\sqrt{2+2 \prod_{j=1}^{n} q_{\alpha_{j}}}}\left(\left|\alpha_{1}, \alpha_{2}, \ldots, \alpha_{n}\right\rangle+\left|-\alpha_{1},-\alpha_{2}, \ldots,-\alpha_{n}\right\rangle\right)
$$

where

$$
q_{\alpha_{j}}=\left\langle\alpha_{j} \mid-\alpha_{j}\right\rangle=\exp \left(-2\left|\alpha_{j}\right|^{2}\right), \quad j=1, \ldots, n .
$$

Let us define two subsystems for the state under consideration: $A$ and $B$. Assume subsystem $A$ contains $n-m$ different coherent modes with parameters $\alpha_{1}, \alpha_{2}, \ldots, \alpha_{n-m}$ (for example, the first $n-m$ modes), and subsystem $B$ comprises $m$ modes with coherence parameters $\alpha_{n-m+1}, \alpha_{n-m+2}, \ldots, \alpha_{n}$ (for example, the last $m$ modes).

In this study, the following result was obtained: regardless of the dimension of the state, it can be reduced to an effective two-level system. In other words, any quantum Schrödiger cat state of dimension greater than two, consisting of two subsystems, can be associated with the two-mode quantum Schrödiger cat state.

In order to study the correlation and interference properties of subsystems $A$ and $B$, we introduce two coherent states with coherence parameters $a$ and $b$, which are related to the original parameters as follows:

$$
a=\sqrt{\sum_{j=1}^{n-m}\left|\alpha_{j}\right|^{2}}, \quad b=\sqrt{\sum_{j=1}^{m}\left|\alpha_{n-m+j}\right|^{2}} .
$$

Let us write the two-dimensional quantum Schrödiger cat state for these two parameters based on (12):

$$
\left|c a t_{a b}\right\rangle=\frac{1}{\sqrt{2+2 q_{a} q_{b}}}(|a, b\rangle+|-a,-b\rangle) .
$$

It can be shown that for states (17) and (19) the Schmidt numbers exactly coincide (see Appendix). This makes it possible to analyze the coherence between subsystems of arbitrary dimensions.

In addition, for the multidimensional Schrödiger cat states, the interpretation in the form of a doubleslit experiment is also valid. In order to estimate the interference properties, it is necessary to obtain the wave function of state (17) in the momentum representation. This can be done by analogy with formula (14), 

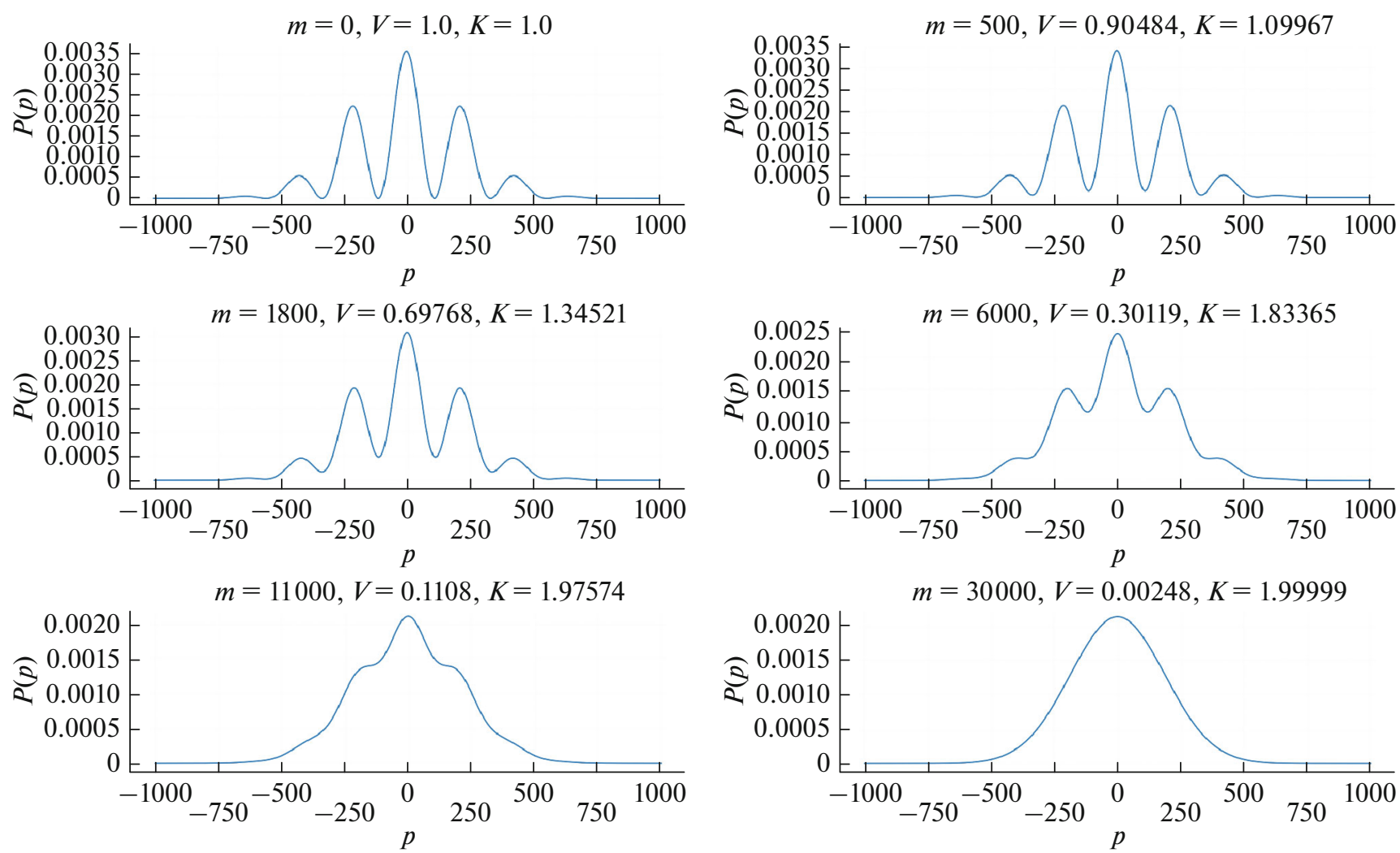

Fig. 3. The gradual disappearance of the interference pattern with an increase in the number of environment modes. The abscissa shows the total momentum $p$.

$$
\tilde{\Psi}_{\alpha_{1} \alpha_{2} \ldots \alpha_{n}}\left(p_{1}, p_{2}, \ldots, p_{n}\right)=\frac{\sqrt{2} \tilde{C}_{\alpha_{1} \alpha_{2} \ldots \alpha_{n}}}{\sqrt[4]{\pi^{n}}} \exp \left(-\frac{1}{2} \sum_{j=1}^{n} p_{j}^{2}\right) \cos \left(\sqrt{2} \sum_{j=1}^{n} \alpha_{j} p_{j}\right),
$$

where the normalization factor is given by the formula

$$
\tilde{C}_{\alpha_{1} \alpha_{2} \ldots \alpha_{n}}=\left(\exp \left(2 \sum_{j=1}^{n} \overline{\bar{\alpha}}_{j}^{2}\right)+\exp \left(-2 \sum_{j=1}^{n} \bar{\alpha}_{j}^{2}\right)\right)^{-1 / 2},
$$

and the real and imaginary parts of the amplitudes of the coherent states are $\bar{\alpha}_{j}=\operatorname{Re}\left(\alpha_{j}\right)$ and $\overline{\bar{\alpha}}_{j}=\operatorname{Im}\left(\alpha_{j}\right)$.

Next, we find the corresponding probability distribution and integrate over the variables corresponding to the environment (the last $m$ modes). The resulting marginal distribution of the first $n-m$ variables will describe the corresponding multidimensional interference pattern

$$
\begin{aligned}
\tilde{P}_{\alpha_{1} \alpha_{2} \ldots \alpha_{n}}\left(p_{1}, p_{2}, \ldots, p_{n-m}\right) & =\frac{\tilde{C}_{\alpha_{1} \alpha_{2} \ldots \alpha_{n}}^{2}}{\sqrt{\pi^{n-m}}} \exp \left(-\sum_{j=1}^{n-m} p_{j}^{2}\right) \cdot\left[\exp \left(-2 \sum_{j=n-m+1}^{n} \bar{\alpha}_{j}^{2}\right) \cos \left(2 \sqrt{2} \sum_{j=1}^{n-m} \bar{\alpha}_{j} p_{j}\right)\right. \\
& \left.+\exp \left(2 \sum_{j=n-m+1}^{n} \overline{\bar{\alpha}}_{j}^{2}\right) \cosh \left(2 \sqrt{2} \sum_{j=1}^{n-m} \overline{\bar{\alpha}}_{j} p_{j}\right)\right] .
\end{aligned}
$$

For example, let us take a 100000 -dimensional Schrödiger cat state with parameters $\alpha_{1}=\alpha_{2}=\ldots=\alpha_{n}=\alpha=0.01$ and consider the gradual disappearance of interference as the number of modes considered as the environment increases (see Fig. 3).
Let us introduce the total momentum of the system related to the interference pattern:

$$
p=p_{1}+\ldots+p_{n-m} .
$$

In the case when parameter $\alpha$ is valid, we can get the following expression for the distribution of the interfering variable $p$ : 


$$
\tilde{P}(p)=\frac{1}{\left(1+\exp \left(-2 n \alpha^{2}\right)\right) \sqrt{\pi(n-m)}} \exp \left(-\frac{p^{2}}{n-m}\right)[1+V \cos (2 \sqrt{2} \alpha p)] \text {. }
$$

Here parameter $V$ is the visibility of the interference pattern in the approximation of clearly distinguishable alternatives. It is determined by the number of the environment's modes $m$ and is given by the following simple formula:

$$
V=\exp \left(-2 m \alpha^{2}\right) \text {. }
$$

The obtained dependences show that the visibility of the interference pattern sharply decreases even with the reduction of one photon in the modes of the environment. For example using $m=11000$ modes as the environment corresponds to the reduction of 1.1 photon (since in the example under consideration the average number of photons per mode is $|\alpha|^{2}=0.0001$ ).

The obtained analytical formulas for the multidimensional Schrödiger cat state of arbitrary dimensions make it possible to analyze the correlational characteristics using the Schmidt decomposition and estimate the parameters of interference double-slit experiments with varying degrees of influence of the environment.

\section{CONCLUSIONS}

In this study, a mathematical apparatus was developed for systems with interfering alternatives. As an example of such systems, the quantum Schrödiger cat states and the wave functions of the double-slit experiment were considered. These systems turned out to be similar in structure, which makes it easy to move from one consideration to another. The resulting relationship allows us to efficiently calculate various interference characteristics, such as visibility and coherence.

It was shown that, from the quantum information point of view, the complex parameter of the coherence of light oscillations, which is important for optics, is naturally related to the scalar product of the states of the environment corresponding to the interfering alternatives. A simple explicit formula was obtained that sets the visibility of the interference pattern depending on the Schmidt number, which characterizes the degree of correlation between the interfering system and its environment.

The results of the study are generalized to the case of the Schrödiger cat states of arbitrary dimensions. Explicit formulas are obtained for the reduced states arising in the measurement of some of the modes of the considered multimode system.

The results of the research carried out have significant applied value and can be used in the development of highdimensional quantum information processing systems.

\section{FUNDING}

The work was carried out within the framework of the State assignment of the Valiev IPT RAS, Ministry of Science and Higher Education of the Russian Federation on topic no. 0066-2019-0005 and supported by the Fund for the Development of Theoretical Physics and Mathematics "BAZIS", grant no. 20-1-1-34-1.

\section{CONFLICT OF INTEREST}

The authors declare that they have no conflict of interest.

\section{OPEN ACCESS}

This article is licensed under a Creative Commons Attribution 4.0 International License, which permits use, sharing, adaptation, distribution and reproduction in any medium or format, as long as you give appropriate credit to the original author(s) and the source, provide a link to the Creative Commons license, and indicate if changes were made. The images or other third party material in this article are included in the article's Creative Commons license, unless indicated otherwise in a credit line to the material. If material is not included in the article's Creative Commons license and your intended use is not permitted by statutory regulation or exceeds the permitted use, you will need to obtain permission directly from the copyright holder. To view a copy of this license, visit http://creativecommons.org/licenses/by/4.0/.

The statement on the equality of the Schmidt numbers of formulas (17) and (19) follows from the equivalence of effective two-level systems. Let us first consider formula (19), which is exactly equivalent to (12):

$$
\left|c a t_{\alpha \beta}\right\rangle=\frac{1}{\sqrt{2+2 q_{\alpha} q_{\beta}}}(|\alpha, \beta\rangle+|-\alpha,-\beta\rangle) \text {. }
$$

The corresponding effective two-level system is determined by replacing it with the orthogonal qubit states (4) and (5). In this case, 


$$
\begin{aligned}
& |0\rangle_{1}=|\alpha\rangle, \quad|1\rangle_{1}=\frac{1}{\sqrt{1-q_{\alpha}^{2}}}\left(|-\alpha\rangle-q_{\alpha}|\alpha\rangle\right), \\
& |0\rangle_{2}=|\beta\rangle, \quad|1\rangle_{2}=\frac{1}{\sqrt{1-q_{\beta}^{2}}}\left(|-\beta\rangle-q_{\beta}|\beta\rangle\right),
\end{aligned}
$$

where $q_{\alpha}=\exp \left(-2|\alpha|^{2}\right)$ and $q_{\beta}=\exp \left(-2|\beta|^{2}\right)$. Then the two-dimensional Schrödiger cat state can be represented as a two-qubit state:

$$
\left|c a t_{\alpha \beta}\right\rangle=\frac{1}{\sqrt{2+2 q_{\alpha} q_{\beta}}}\left(\left(1+q_{\alpha} q_{\beta}\right)|00\rangle+q_{\alpha} \sqrt{1-q_{\beta}^{2}}|01\rangle+q_{\beta} \sqrt{1-q_{\alpha}^{2}}|10\rangle+\sqrt{1-q_{\alpha}^{2}} \sqrt{1-q_{\beta}^{2}}|11\rangle\right) .
$$

Let us now consider the formula for the multidimensional Schrödiger cat state (17).

$$
\left|c a t_{\alpha_{1} \alpha_{2} \ldots \alpha_{n}}\right\rangle=\frac{1}{\sqrt{2+2 \prod_{j=1}^{n} q_{\alpha_{j}}}}\left(\left|\alpha_{1}, \alpha_{2}, \ldots, \alpha_{n}\right\rangle+\left|-\alpha_{1},-\alpha_{2}, \ldots,-\alpha_{n}\right\rangle\right) .
$$

An effective two-qubit system can be defined by analogy with the two-dimensional Schrödiger cat state. For this, it is necessary to take into account that subsystem $A$ consists of the first $n-m$ modes $\left(\alpha_{1}, \alpha_{2}, \ldots, \alpha_{n-m}\right)$, and subsystem $B$ consists of the remaining $m$ modes $\left(\alpha_{n-m+1}, \alpha_{n-m+2}, \ldots, \alpha_{n}\right)$. Next, we need to make the following replacements:

$$
\begin{gathered}
|0\rangle_{1}=\left|\alpha_{1}, \alpha_{2}, \ldots, \alpha_{n-m}\right\rangle, \\
|1\rangle_{1}=\frac{1}{\sqrt{1-\prod_{i=1}^{n-m} q_{\alpha_{i}}^{2}}}\left(\left|-\alpha_{1},-\alpha_{2}, \ldots,-\alpha_{n-m}\right\rangle-\prod_{i=1}^{n-m} q_{\alpha_{i}}\left|\alpha_{1}, \alpha_{2}, \ldots, \alpha_{n-m}\right\rangle\right) \\
|1\rangle_{2}=\frac{1}{\sqrt{1-\prod_{i=1}^{m} q_{\alpha_{n-m+i}}^{2}}}\left(\left|-\alpha_{n-m+1},-\alpha_{n-m+2}, \ldots,-\alpha_{n}\right\rangle-\prod_{i=1}^{m} q_{\alpha_{n-m+i}}\left|\alpha_{n-m+1}, \alpha_{n-m+2}, \ldots, \alpha_{n}\right\rangle\right)
\end{gathered}
$$

Then the formula for the multidimensional Schrödiger cat state can be represented as

$$
\begin{gathered}
\left|c a t_{\alpha_{1} \alpha_{2} \ldots \alpha_{n}}\right\rangle=\frac{1}{\sqrt{2+2 \prod_{j=1}^{n} q_{\alpha_{j}}}\left(\left(1+\prod_{i=1}^{n} q_{\alpha_{i}}\right)|00\rangle+\prod_{i=1}^{n-m} q_{\alpha_{i}} \sqrt{1-\prod_{i=1}^{m} q_{\alpha_{n-m+i}}^{2}}|01\rangle\right.} \\
\left.+\prod_{i=1}^{m} q_{\alpha_{n-m+i}} \sqrt{1-\prod_{i=1}^{n-m} q_{\alpha_{i}}^{2}}|10\rangle+\sqrt{1-\prod_{i=1}^{n-m} q_{\alpha_{i}}^{2}} \sqrt{1-\prod_{i=1}^{m} q_{\alpha_{n-m+i}}^{2}}|11\rangle\right) .
\end{gathered}
$$

It can be noted that the structure of this formula is identical to formula (25). If we introduce replacement (18), then the amplitudes of the basic states are simplified:

$$
\begin{gathered}
\prod_{i=1}^{n-m} q_{\alpha_{i}}=\prod_{i=1}^{n-m} \exp \left(-2\left|\alpha_{i}\right|^{2}\right)=\exp \left(-2 \sum_{i=1}^{n-m}\left|\alpha_{i}\right|^{2}\right)=\exp \left(-2 a^{2}\right)=q_{a}, \\
\prod_{i=1}^{m} q_{\alpha_{n-m+i}}=\prod_{i=1}^{m} \exp \left(-2\left|\alpha_{n-m+i}\right|^{2}\right)=\exp \left(-2 \sum_{i=1}^{m}\left|\alpha_{n-m+i}\right|^{2}\right)=\exp \left(-2 b^{2}\right)=q_{b} .
\end{gathered}
$$

As a result, we find that formula (26) exactly transforms into formula (25). The equivalence of formulas (25) and (26) indicates the equality of all the characteristics that are calculated using the corresponding qubit representation, including the Schmidt coefficients, the Schmidt number, and the visibility of the interference pattern. 


\section{REFERENCES}

1. Valiev, K.A. and Kokin, A.A., Kvantovye komp'yutery: nadezhda i real'nost' (Quantum Computers: Hope and Reality), Izhevsk: RKhD, 2001.

2. Nielsen, M. and Chuang, I., Quantum Computation and Quantum Information, Cambridge: Cambridge Univ., 2000.

3. Buks, E., Schuster, R., Heiblum, M., Mahalu, D., and Umansky, V., Dephasing in electron interference by a 'which-path' detector, Nature (London, U.K.), 1998, vol. 391, pp. 871-874.

4. Glauber, R.J., Coherent and incoherent states of the radiation field, Phys. Rev., 1963, vol. 131, p. 2766.

5. Van Enk, S.J. and Fuchs, C.A., Quantum state of an ideal propagating laser field, Phys. Rev. Lett., 2001, vol. 88, p. 027902.

6. Tan, K.C., Volkoff, T., Kwon, H., and Jeong, H., Quantifying the coherence between coherent states, Phys. Rev. Lett., 2017, vol. 119, p. 190405.

7. Bose, S., Home, D., and Mal, S., Nonclassicality of the harmonic-oscillator coherent state persisting up to the macroscopic domain, Phys. Rev. Lett., 2018, vol. 120, p. 210402.

8. Neergaard-Nielsen, J.S., Takeuchi, M., Wakui, K., Takahashi, H., Hayasaka, K., Takeoka, M., and Sasaki, M., Optical continuous-variable qubit, Phys. Rev. Lett., 2010, vol. 105, p. 053602.

9. Ralph, T.C., Gilchrist, A., Milburn, G.J., Munro, W.J., and Glancy, S., Quantum computation with optical coherent states, Phys. Rev. A, 2003, vol. 68, p. 042319.

10. Jeong, H. and Kim, M.S., Efficient quantum computation using coherent states, Phys. Rev. A, 2002, vol. 65, p. 042305.

11. Cochrane, P.T., Milburn, G.J., and Munro, W.J., Macroscopically distinct quantum-superposition states as a bosonic code for amplitude damping, Phys. Rev. A, 1999, vol. 59, p. 2631.

12. Gottesman, D., Kitaev, A., and Preskill, J., Encoding a qubit in an oscillator, Phys. Rev. A, 2001, vol. 64, p. 012310.

13. Ralph, T.C., Coherent superposition states as quantum rulers, Phys. Rev. A, 2002, vol. 65, p. 042313.

14. Joo, J., Munro, W.J., and Spiller, T.P., Quantum metrology with entangled coherent states, Phys. Rev. Lett., 2011, vol. 107, p. 083601.

15. Bartlett, S.D. and Sanders, B.C., Universal continuous-variable quantum computation: Requirement of optical nonlinearity for photon counting, Phys. Rev. A, 2002, vol. 65, p. 042304.

16. Daoud, M. and Choubabi, E.B., Bipartite entanglement of multipartite coherent states using quantum network of beam splitters, Int. J. Quantum Inform., 2012, vol. 10, p. 1250009.

17. Jeong, H. and An, N.B., Greenberger-HorneZeilinger-type and W-type entangled coherent states: Generation and Bell-type inequality tests without photon counting, Phys. Rev. A, 2006, vol. 74, p. 022104.

18. Munhoz, P.P., Semião, F.L., Vidiella-Barranco, A., and Roversi, J.A., Cluster-type entangled coherent states, Phys. Lett. A, 2008, vol. 372, pp. 3580-3585.

19. Bogdanov, A.Yu., Bogdanov, Yu.I., and Valiev, K.A., Schmidt information and entanglement of quantum systems, Mosc. Univ. Comput. Math. Cybern., 2007, vol. 31, no. 1, pp. 33-42.

20. Bogdanov, Yu.I., Fastovets, D.V., Bogdanova, N.A., Lukichev, V.F., and Chernyavskii, A.Yu., Schmidt decomposition and analysis of statistical correlations, Russ. Microelectron., 2016, vol. 45, no. 5, pp. 314-323.

21. Bogdanov, Yu.I., Fundamental notions of classical and quantum statistics: A root approach, Opt. Spectrosc., 2004, vol. 96, no. 5, pp. 668-678.

22. Bogdanov, Yu.I., Valiev, K.A., Nuyanzin, S.A., and Gavrichenko, A.K., Information aspects of 'whichpath' interference experiments with microparticles, Russ. Microelectron., 2010, vol. 39, no. 4, pp. 221-242.

23. Bogdanov, Yu.I., Bogdanov, A.Yu., Nuianzin, S.A., and Gavrichenko, A.K., On the informational aspects of interfering quantum states, arXiv: 0812.4808 [quant-ph].

24. Zych, M., Costa, F., Pikovski, I., and Brukner, C., Quantum interferometric visibility as a witness of general relativistic proper time, Nat. Commun., 2011, vol. 2, p. 505.

25. Born, M. and Wolf, E., Principles of Optics, Oxford: Pergamon, 1964.

26. Dodonov, V.V., Malkin, I.A., and Man'ko, V.I., Even and odd coherent states and excitations of a singular oscillator, Physica (Amsterdam, Neth.), 1974, vol. 72, no. 3, pp. 597-615.

27. Glancy, S. and Vasconcelos, H.M., Methods for producing optical coherent state superpositions, J. Opt. Soc. Am. B, 2008, vol. 25, pp. 712-733.

28. Gilchrist, A., Nemoto, K., Munro, W.J., Ralph, T.C., Glancy, S., Braunstein, S.L., and Milburn, G.J., Schrödinger cats and their power for quantum information processing, J. Opt. B: Quantum Semiclass. Opt., 2004, vol. 6, no. 8, pp. S828-S833.

29. Daoud, M., Laamara, A.R., and Essaber, R., Quantum correlations dynamics of quasi-bell cat states, Int. J. Quantum Inform., 2013, vol. 11, no. 6, p. 1350057.

30. Feynmann, R. and Hibbs, A., Quantum Mechanics and Path Integrals, New York: Dover, 2010. 